\title{
An analog optical link in surface mount technology (SMT) for multichannel biomedical data acquisition
}

\author{
WOLFGANG TEDER \\ University of Helsinki, Helsinki, Finland
}

\begin{abstract}
A stand-alone, low-cost analog optical link for biomedical data is described. This compact device features low power consumption and low noise and serves as a safe link between biodata amplifiers and computerized recording equipment. A modular surface mount technology design assures the high reliability and high package density required in multichannel applications in EEG and event-related potential research. With only a few modifications, the data link can transmit dc signals with low drift, or interface fast signals such as brainstem evoked potentials. The maximum bandwidth ranges from dc to $5000 \mathrm{~Hz}$.
\end{abstract}

For economical reasons, optically isolated multichannel data acquisition usually involves a multiplexer-demultiplexer (MUX-DEMUX) switching circuitry and a serial digital optical link in between. The incoming ana$\log$ input voltages are sequentially sampled, and a subsequent voltage-to-frequency converter feeds a pulsewidth-modulated (PWM) optical link. Associated digital circuitry synchronously controls MUX and DEMUX switches and provides a transmission protocol for proper decoding of output channels. The transmission performance of such a system is very linear, because only highlevel, digital pulse trains are transmitted via the optical fiber. This approach is very economical, because many channels are sequentially fed through one (high-speed) optical link. However, when maximum flexibility is required, a "closed" MUX-DEMUX system can become a bottleneck in data acquisition, as can the considerable circuit complexity for 32 or more channels, for at least two reasons.

First, some advanced paradigms in event-related potential (ERP) research ideally require parallel data acquisition of slowly varying $(\mathrm{dc})$ and high-frequency signals (e.g., brainstem responses). In a MUX-DEMUX system, the clock speed leads to an equal sample time common for all channels and determines the available bandwidth. Because all the channels have identical bandwidths, it is almost impossible to customize the parameters of a small subset of channels according to different recording requirements. In other words, such a system is highly impractical for recording qualitatively different biodata that require specifically tailored settings. Sec-

This work was partly supported by the Academy of Finland. Correspondence should be addressed to W. Teder, Cognitive Psychology Research Unit, Department of Psychology, Ritarikatu 5, University of Helsinki, SF-00014 Helsinki, Finland (e-mail: wolfgang.teder(a) helsinki.fi). ond, the serial data transmission in a MUX-DEMUX system requires decoding for signal restoration and makes it difficult to flexibly establish multiple connections to auxiliary devices (e.g., EEG paper recorder, oscilloscope, on-line monitoring systems).

In contrast, parallel data transmission does not have the aforementioned limitations, because peripheral devices can be connected easily by nontechnical operators. However, a fully digital multichannel version is not very cost effective, since each channel requires its own voltage-tofrequency converter and PWM link. An alternative lowcost approach is to use parallel analog data links with negligible sacrifice of data quality. In ERP research, it is unrealistic to make exaggerated demands on parameters such as transmission linearity and noise. In most applications, rather, the "physiological noise" (such as eye movements, slow shifts, muscle artifacts, etc.) and electrode shifts dominate the error budget of the recording system.

\section{BACKGROUND}

Special optocouplers for analog data interfacing, such as the IL 300 (Siemens), provide an internal insulation distance of $>0.4 \mathrm{~mm}$ and safely withstand voltages of about $4.5 \mathrm{kV}$ ac $\mathrm{RMS}_{\mathrm{RMS}}$ between input and output pins. Although these features should guarantee patient security, some countries and research institutes demand even higher insulation voltages well exceeding $5 \mathrm{kV}$ for custom-made medical equipment. Therefore, we selected industrial standard optocouplers (CNY 65B, Telefunken), developed and approved for high isolation voltages of $11.6 \mathrm{kVdc}{ }^{1}$

A typical servo circuit utilizes a well-matched pair of optocouplers for OC1 and OC2 and needs equal power supplies for both sides of the data link (Figure 1). Under the condition that $\mathrm{OC} 1$ and $\mathrm{OC} 2$ track each other over a defined excursion range, the output of amplifier $U 2$ will 


\section{SERUOCIRCUIT FOR ANALOG TRANSMISSION}

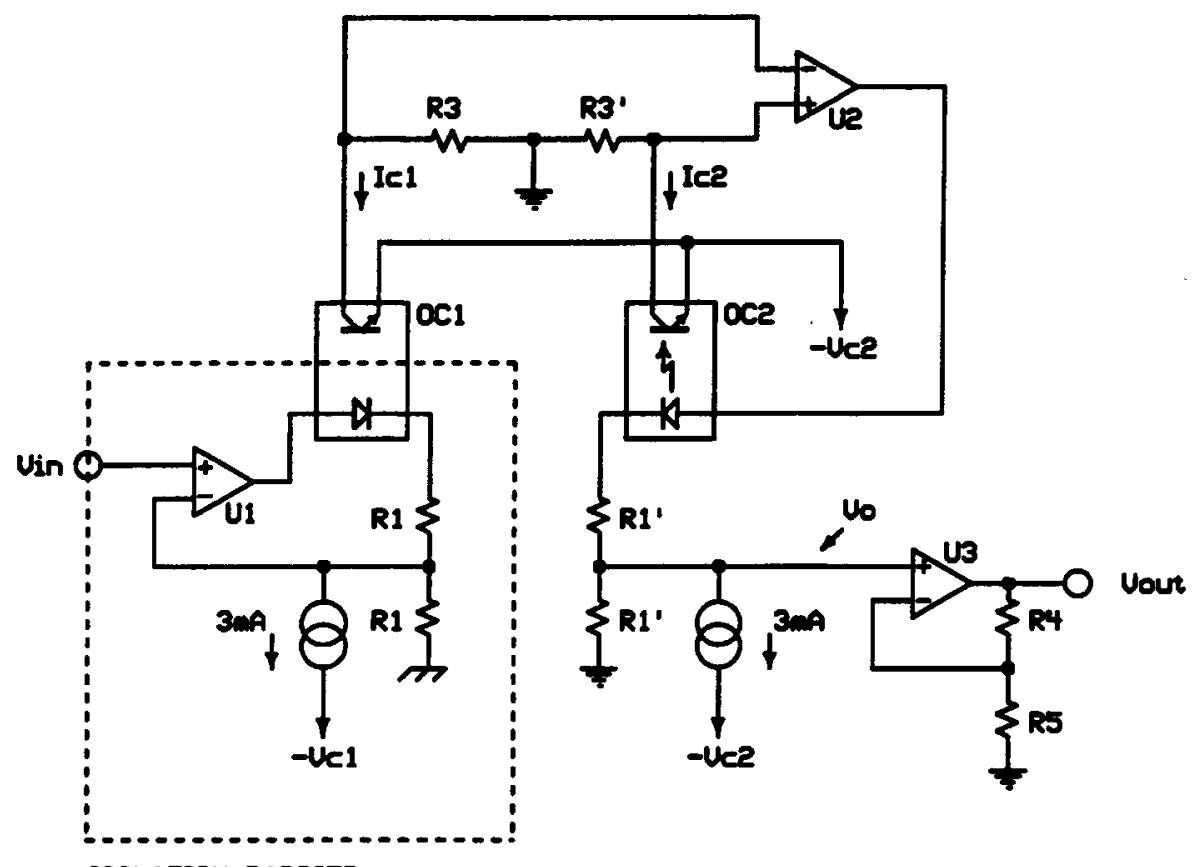

ISOLATION BARRIER

Figure 1. Schematic diagram of servocircuit. Assuming a matched pair for optocouplers $O C 1$ and $O C 2$, the output of amplifier $U 2$ will force the currents $I_{L E D} 2$ and $I_{L E D} 1$ to be equal. Given very stable power supplies for both sides, the $3 \mathrm{~mA}$ constant-current sources can be replaced by resistors with little sacrifice in performance. Resistors $R 4$ and $R 5$ are dimensioned for the desired output swing required by the AD converter.

force the currents $\mathrm{Ic}_{1}$ and $\mathrm{Ic}_{2}$ to be equal. ${ }^{2}$ Therefore, the nonlinearities tend to cancel, and the overall transfer function will be almost perfectly linear. In principle, a servo solution guarantees improved transmission linearity over simpler approaches using a single light-emitting diode (LED) biased at midpoint (Fridlund, Price, \& Fowler, 1982).

Through the use of precision amplifiers and highquality passive components, a sufficient linearity of $\geq 99.5 \%$ at $2-V_{p p}$ output swing can easily be obtained with dual optocouplers - such as the HCPL 2530 type from Hewlett-Packard (see Precision Monolithics, 1987).

For a performance similar to that of dual optocouplers, matched pairs from single units have to be selected. Unfortunately, optocouplers show substantial variations in initial parameters, this variation being even worse for units coming from different production runs. In Figure 2 , we have plotted the output offset voltage (obtained with a typical burn-in circuit) for a total of 97 units from three different production runs. Some couplers can have up to $20 \mathrm{mV}$ differences in offset voltage. Owing to this fact, all couplers used for the assembly of a multichannel device should be selected from the same production charge.

Apart from initial tolerances, it can easily be demonstrated that optocoupler parameters are subject to drift with temperature and physical age. In this particular application, however, temperature drift is only a small problem. First, ambient temperature in a typical medical laboratory environment fluctuates only within a narrow range of about $\pm 2^{\circ} \mathrm{C}$. Second, in a servo linearizer cir-
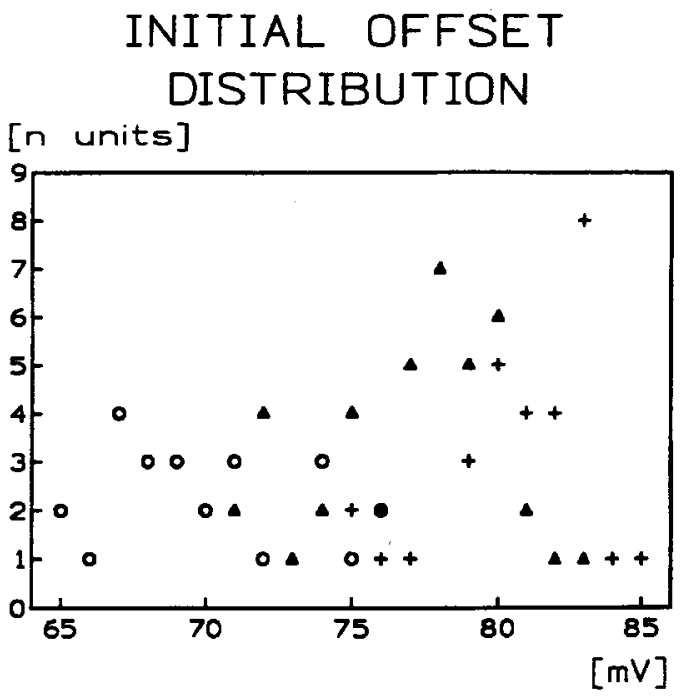

Figure 2. Variation of offset voltage in three different production runs of the type CNY-65B. 


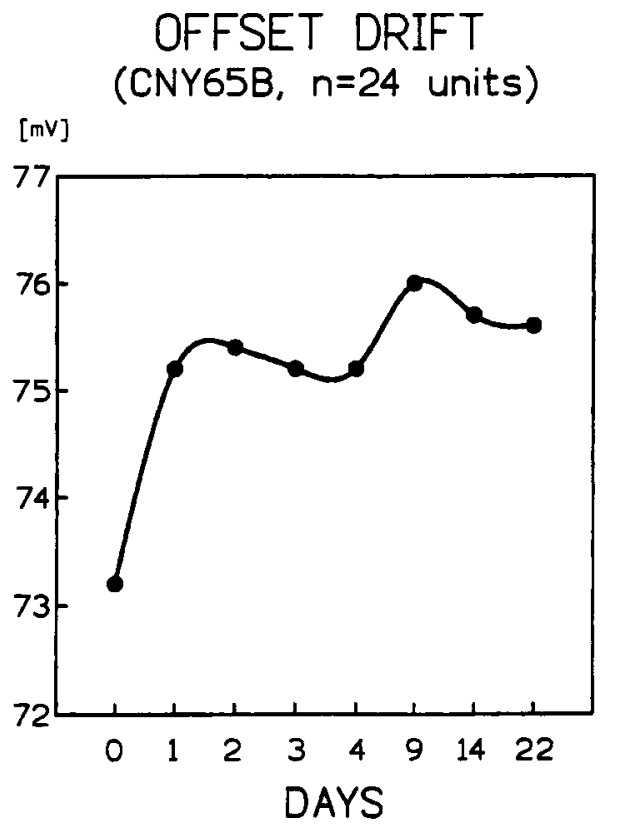

Figure 3. Variation of offset voltage during a burn-in period of about 3 weeks.

cuit like that in Figure 1, temperature changes will influence a matched pair similarly, and thus tend to cancel.

The offset drift over time is a more critical design parameter, especially in optical links customized for transmission of dc signals. In Figure 3, we have plotted the average offset variation of 24 units over a period of 3 weeks. The burn-in procedure was done with constant- current sources adjusted to $5.0 \mathrm{~mA}$. The average offset variation over time was tested by means of the mean square successsive difference test (Hald, 1952). If the offset drift is not under statistical control, cyclical fluctuations must be expected. The observed value of $r=$ -1.56 deviated significantly $(p \leq .01)$ from the expected value $(r=.393)$. Hence, the offset drift was not under statistical control but rather was determined by either the manufacturing process itself or conditions under which the measurements were taken. However, excluding for the initial measurement (Day 0), the remaining drift neither differed significantly from zero nor departed from linearity, as confirmed by a regression analysis $[F(1,5)=3.22, p=.13]$ and a run test $(p=$ .54 ), respectively. The $95 \%$ confidence interval for the time period spanning from Day 1 to Day 22 ranged from $74.5 \mathrm{mV}$ to $75.6 \mathrm{mV}$. Therefore, we decided to select matched pairs with similar output offset $(\leq 0.5 \mathrm{mV})$ for the final circuit (Figure 4) after a burn-in period of 2 days. This procedure aims at a very linear performance and is strongly recommended for research applications. Should the unit be used in a teaching laboratory, a somewhat more relaxed selection procedure $( \pm 2.3 \mathrm{mV})$ will usually suffice. With a typical output swing of about $\pm 1 V_{\text {eff }}$, a mismatch of about $10 \mathrm{mV}$ between a given pair of optocouplers will add at least about $1 \%-2 \%$ linearity error to the budget.

\section{DESIGN}

Figure 4 shows a standard ac-coupled channel configuration for EEG recordings (bandpass $0.1-100 \mathrm{~Hz}$, $-3-\mathrm{dB}$ corners). The dashed box on the left contains the

\section{ANALOG OPTOLINK FOR EEG-DATA}

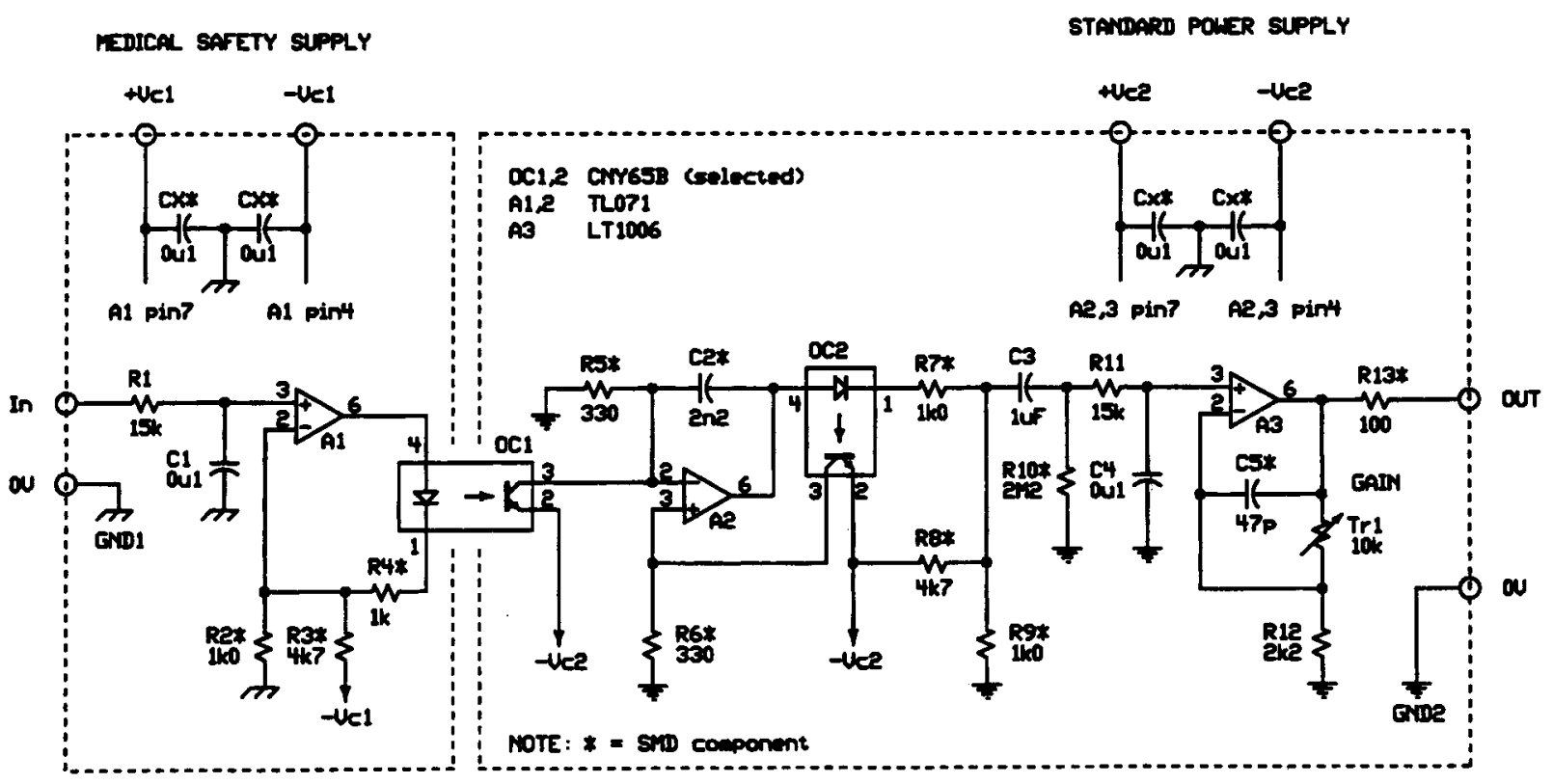

Figure 4. The circuit of the analog data link. 

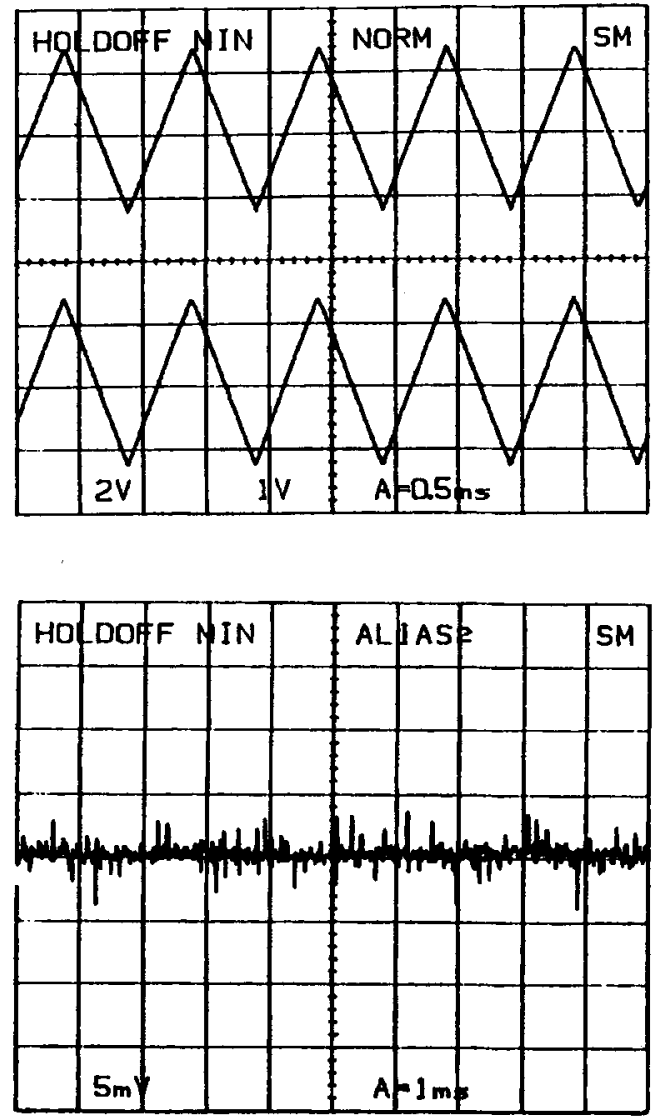

Figure 5. Top: Transmixsion of a 1-kHz triangle wave. Input (top trace, vert. Div. $1 \mathrm{~V} / \mathrm{cm}$ ) and output (bottom trace, vert. Div. $2 \mathrm{~V} / \mathrm{cm}$ ) of optical link (overall circuit gain = 5.0). Bottom: Noise performance with a single-order 10-kHz low pass (hor. Div. $1 \mathrm{msec} / \mathrm{cm}$, vert. Div. $5 \mathrm{mV} / \mathrm{cm})$. Overall circuit gain was adjusted to provide $\pm 5-\mathrm{V}$ output to the $A D$-card with $\pm 1 \mathrm{~V}$ applied to the input of the optolink.

transmitter side of the circuit powered by a medical safety supply or stabilized battery power units. The resistor $\mathrm{R} 1$ and capacitor $\mathrm{C} 1$ form an optional low-pass filter with about $106-\mathrm{Hz}(-3-\mathrm{dB})$ cutoff frequency. The capacitor $\mathrm{C} 2$ in the feedback loop of amplifier A2 is required for stability. Capacitor $\mathrm{C} 3$ and resistor $\mathrm{R} 10$ act as a high-pass filter with a cutoff of about $0.07 \mathrm{~Hz}$. In the ac-coupled mode, the low-noise precision amplifier LT 1006 type (Linear Technology) does not require offset trimming.

For dc applications, however, C3 and R10 should be omitted, and an offset trimpot for amplifier A3 should be connected according to the specifications of the manufacturer. Resistor R11 and capacitor C4 act as an antialiasing filter and determine (as do R1 and C1) the upper frequency limit of the circuit. A maximum bandwidth of dc $5000 \mathrm{~Hz}$ is possible. The overall circuit gain is adjustable with trimpot Trl.

Figure 5 shows the performance data of the circuit depicted in Figure 4. With $1-\mathrm{kHz}$ (1-Veff, triangle wave) input, a superimposition of input and output traces on the oscilloscope did not reveal any visible differences. The bottom trace in Figure 5 shows the worst-case residual wideband noise of the circuit (low-pass filters removed). In a typical EEG recording setup with the $100-\mathrm{Hz}$ lowpass filters installed, the voltage noise at the output is about $2.0 \mathrm{mVeff}$ (worst case). That is, with an output swing of $65 \mathrm{~V}$, the noise floor is down at about $270 \mathrm{~dB}$. This value is more than sufficient for low-noise transmission of EEG data.

Figure 6 shows the board layout (scale 1:2) for the data link. The pads with filled center holes are the solder terminals for the SMT components. Figure 7 depicts the locations of SMT and conventional components on the board. The use of SMT parts assures high reliability and high package density (about 30\%-50\% less board space needed over conventional designs). Only the components that might be subject to change depending on the application are provided in conventional technology. Since all connections are brought to one side of the board, it is convenient to assemble all channel boards on one motherboard. Care should be taken to provide sufficient capacitive decoupling of the supply leads. ${ }^{3}$ Lownoise power supplies for both sides of the link are mandatory. Currently, a total of about 100 units equivalent to the circuit shown in Figure 4 work satisfactorily
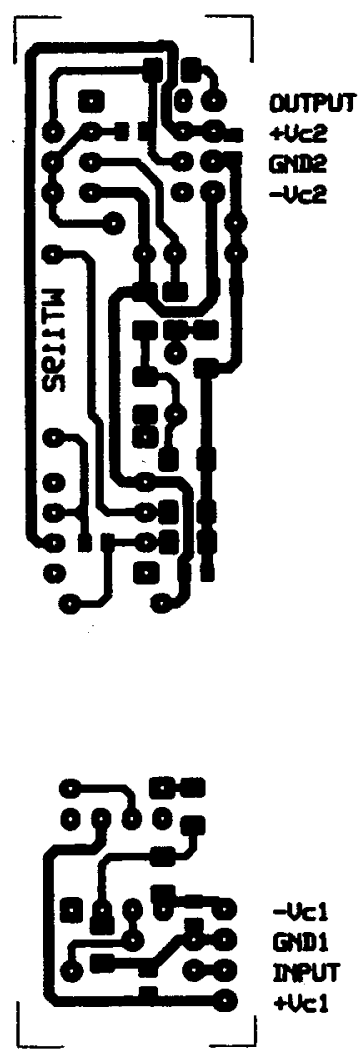

Figure 6. Layout of one channel (1:2), top view of component layer. 


\section{BOARD LAYOUT}

\section{SMD-layer}

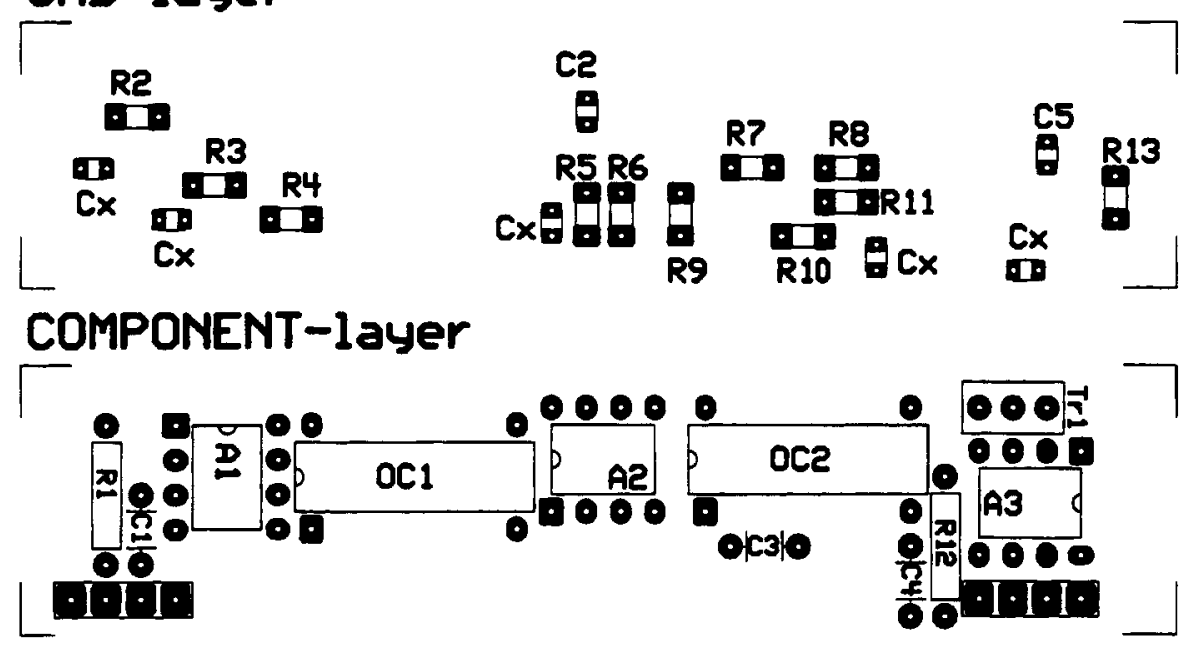

NOTE: both layers top view on component side

Figure 7. Component locations for one channel board.

in different data acquisition systems installed in teaching and research laboratories.

\section{REFERENCES}

Fridlund, A. J., Price, A. W., \& Fowler, S. C. (1982). Low-noise, optically isolated electromyographic preamplifier. Psychophysiology, 19, 701-705.

HALD, A. (1952). Statistical theory with engineering applications. New York: Wiley.

HEWLETT-PACKARD (1987). Linear applications of optocouplers. (Application Note 951-2).

PRECISION Monolithics (1987). Low-bias current, fast J-FET operational amplifier (PMI Application Note OP-43).

SIEMENS. Linear optocoupler IL 300Datasheet.

\section{NOTES}

1. Suitable for SELV (safety extra low voltage): VDE approved according 0883 incl. 0806/IEC 380-0750/IEC 601-0860/IEC 65. ULrecognized: File No. E 76414. Additional VDE approved according 0700 .

2. For a detailed in-depth analysis of transfer functions and nonlinearity errors, see Hewlett-Packard (1987).

3. Interested readers are welcome to ask for more information about the layout and board design from the author. The cost for one channel (excluding the board) is about \$15 US, less for large quantities.

(Manuscript received August 11, 1993; revision accepted for publication January 24,1994 .) 